\title{
A novel water-soluble benzothiazole derivative BD926 triggers ROS-mediated B lymphoma cell apoptosis via mitochondrial and endoplasmic reticulum signaling pathways
}

\author{
MIN-HUI LI ${ }^{1,2^{*}}$, PING YANG ${ }^{3 *}$, TAI YANG $^{2}, \mathrm{KUN}_{\text {ZHANG }}{ }^{4}$, YANG LIU $^{2}$, JIN LIU $^{2}$, \\ LI-MEI LI ${ }^{2}$, XING-YAN LUO ${ }^{2}$, SHU-XIA YANG ${ }^{2}$, QIANG ZOU ${ }^{2}$ and CHONG-JIE ZHANG ${ }^{1}$ \\ ${ }^{1}$ College of Basic and Forensic Medicine, Sichuan University, Chengdu, Sichuan; ${ }^{2}$ Center of Science and Research, \\ ${ }^{3}$ School of Basic Medical Sciences, and ${ }^{4}$ School of Biomedicine Sciences, \\ Chengdu Medical College, Chengdu, Sichuan, P.R. China
}

Received April 5, 2016; Accepted June 22, 2016

DOI: 10.3892/ijo.2016.3684

\begin{abstract}
Benzothiazole derivatives are known for various biological activities, and their potency in cancer therapy have received considerable attention in recent years. However, the poor water solubility of most benzothiazole derivatives has limited their clinical application. We developed BD926, a novel water-soluble benzothiazole derivative and showed here that it could inhibit the proliferation and induce apoptosis of human Ramos B-lymphoma cells. We further showed that BD926 triggered apoptosis through both mitochondria and endoplasmic reticulum pathways. Moreover, BD926 caused cell cycle arrest at G0/G1 stage. Furthermore, accumulation of reactive oxygen species (ROS) were observed after BD926 treatment and ROS inhibitor was able to attenuate BD926induced apoptosis, which suggested that BD926-induced apoptosis may be due to over-producing ROS. These results demonstrate the anticancer effects of BD926 in cell models and raise the possibility for the application of BD926 in cancer therapy.
\end{abstract}

\section{Introduction}

In medicinal chemistry, many compounds with high biological activities derive from scaffolds, and their unique bioactivity may depend on substituent group (1). In addition, designing and transforming the substituent group of small molecule compounds have become an effective way for drug discovery.

Correspondence to: Professor Qiang Zou, Center of Science and Research, Chengdu Medical College, Chengdu, Sichuan, P.R. China E-mail: qiangzou99@gmail.com

Professor Chong-Jie Zhang, College of Basic and Forensic Medicine, Sichuan University, Chengdu, Sichuan, P.R. China

E-mail: cjzhang49@hotmail.com

*Contributed equally

Key words: benzimidazole derivative, B lymphoma, apoptosis, reactive oxygen species
Benzothiazole is a heterocyclic scaffold with a benzene ring fused with a five-membered thiazole ring, and it is recognized as an important basis of the nucleus for drug synthesis $(2,3)$. Benzothiazole derivatives have a wide range of biological activities (4), such as anticancer $(5,6)$, antimicrobial (7) and immunosuppressive activity (8), benzothiazole analogues are also biologically active compounds in the central nervous system (9). In addition, benzothiazole derivatives as commercial drugs are extensively used in the clinical treatment of numerous diseases $(10,11)$.

Our research team first reported a novel benzothiazole derivative BD750 [2-(2-benzothiazoleyl)-4,5,6,7-tetrahydro2H-indazol-3-ol, C14H13N3OS, MW: 271.3]. BD750 has significantly shown immunosuppressive activity by inhibiting $\mathrm{T}$ cell proliferation (8). However, its poor solubility in water is unsatisfactory. In addition, the poor water solubility may make it difficult in clinical application. Thus, our research team was committed to design, synthesize, screen and biological evaluate of novel water-soluble benzothiazole derivatives based on BD750. Then we find BD926, a new water-soluble benzothiazole derivative in which the $\mathrm{H}-2$ of benzothiazol is replaced by 4,5,6,7-tetrahydro-2H-indazol-3-olate group and it has shown good water solubility of approximately $25 \mathrm{mg} / \mathrm{ml}$ in water (12).

In the present study, we investigated the antitumor biological activity of BD926 in the human Ramos B-lymphoma cell line, and explored its potential mechanisms. Our findings aim to clarify that BD926 may be a potential antitumor drug.

\section{Materials and methods}

Drug and reagents. BD926, Sodium 2-(2-benzothiazoleyl)4,5,6,7-tetrahydro-2H-indazol-3-olate (Fig. 1A), was synthesized previously by our team and the structure was confirmed by $1 \mathrm{H}-\mathrm{NMR}, 13 \mathrm{C}-\mathrm{NMR}$ and HRMS (ESI). Purity (85\%) was measured by HPLC analysis (12). BD926 was dissolved in ultrapure water at a stock concentration of $10 \mathrm{mM}$ and stored at $-20^{\circ} \mathrm{V}$.

The antibodies against GAPDH, PARP, Bcl-xl, Bcl-2, Bid, caspase-3, caspase-8, caspase- 9 were purchased from 
A
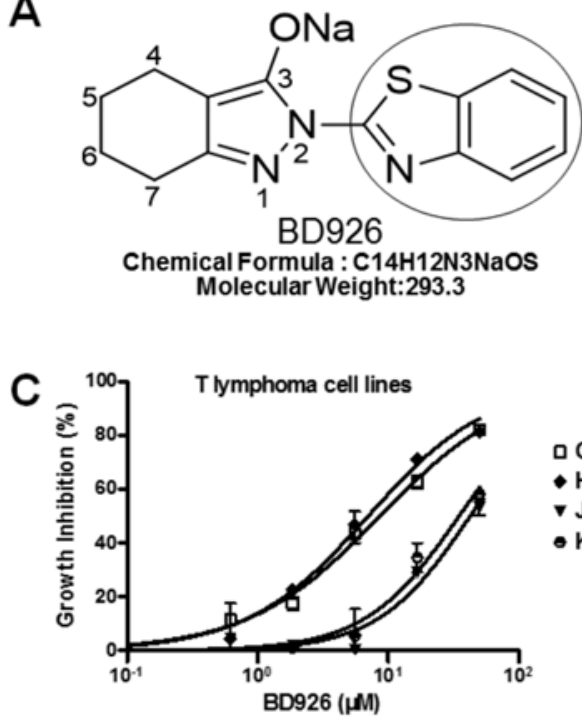

E

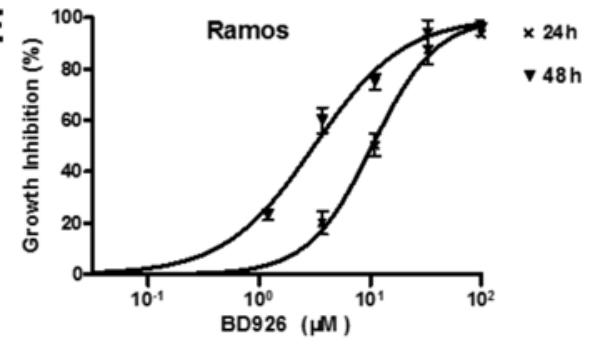

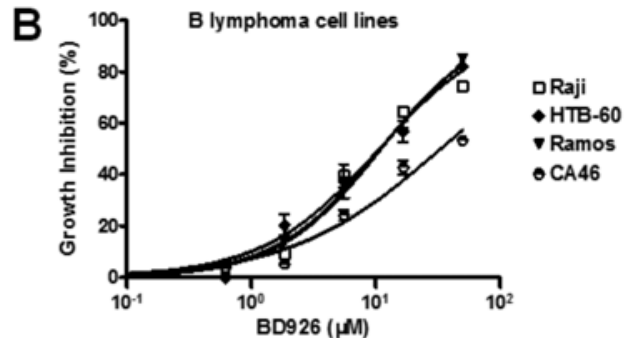

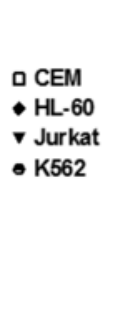

D

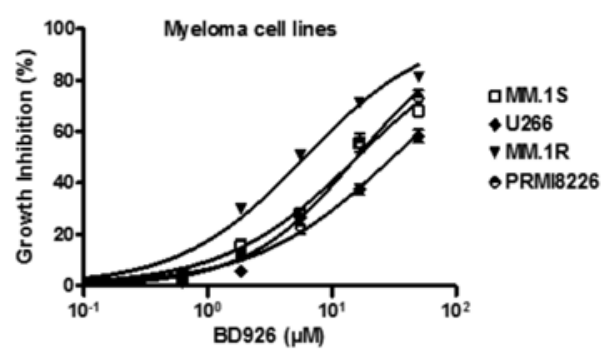

$\mathbf{F}$

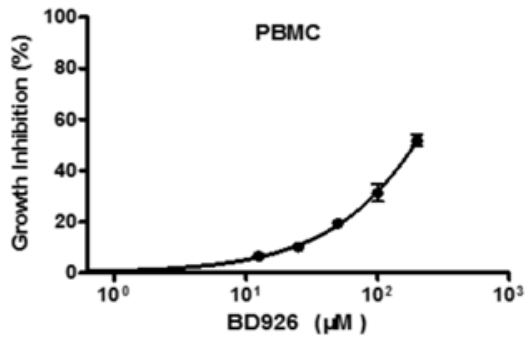

Figure 1. BD926 inhibits proliferation of PBMC and tumor cells in vitro. The growth inhibition effects of BD926 in PBMC and tumor cells were determined by CCK-8 assay. (A) Molecular structure of BD926, in which the H-2 of benzothiazol was replaced by 4,5,6,7-tetrahydro-2H-indazol-3-olate group. (B) BD926 inhibited the proliferation of B lymphoma cell lines in a dose-dependent manner with $\mathrm{IC}_{50} \sim 10.9 \mu \mathrm{M}$ in Ramos, $10.8 \mu \mathrm{M}$ in Raji, $10.7 \mu \mathrm{M}$ in HTB-60 and $33.2 \mu \mathrm{M}$ in CA46. (C) BD926 inhibited the proliferation of T lymphoma cell lines in a dose-dependent manner with IC S0 $_{5} \sim 8.6 \mu \mathrm{M}$ in CEM, 7.0 $\mu \mathrm{M}$ in HL-60, $42.2 \mu \mathrm{M}$ in Jurkat and 34.9 $\mu \mathrm{M}$ in K562. (D) BD926 inhibited the proliferation of myeloma cell lines in a dose-dependent manner with IC $50 \sim 15.9 \mu \mathrm{M}$ in MM.1S, $6 \mu \mathrm{M}$ in MM.1R, 30.9 $\mu \mathrm{M}$ in U266 and 15.8 $\mu \mathrm{M}$ in PRMI-8226. (E) BD926 inhibited the proliferation of Ramos cells in a time- and dose-dependent manner with $\mathrm{IC}_{50} \sim 10.9 \mu \mathrm{M}$ for $24 \mathrm{~h}$ and $3 \mu \mathrm{M}$ for $48 \mathrm{~h}$. (F) BD926 exhibited low toxicity in PBMC with $\mathrm{CC}_{50} \sim 200 \mu \mathrm{M}$ for $48 \mathrm{~h}$. The results are presented as the means $\pm \mathrm{SD}(\mathrm{n}=3)$ and were analyzed with the GraphPad Prism software.

Cell Signaling Technology. The caspase-12 antibody was purchased from Abcam. Primers were synthesized by the Shanghai Biological Engineering Company of China. All other chemicals were of analytical grade.

Cell culture. B lymphoma cell lines Ramos, Raji, HTB-60 and CA46, T lymphoma cell lines CEM, HL-60, Jurkat and K562, myeloma cell lines MM.1S, MM.1R, U266 and PRMI-8226 were saved by our laboratory. The cells were propagated under humidified conditions with $5 \% \mathrm{CO}_{2}$ at $37^{\circ} \mathrm{C}$ in RPMI1640 medium and supplemented with $10 \%$ fetal bovine serum (FBS; both from Gibco, Gaithersburg, MD, USA), 100 units/ml penicillin and streptomycin. To ensure that there was no mycoplasma contamination present, antibiotic treatment was performed regularly.

Human peripheral blood mononuclear cells (PBMC) were isolated from three healthy donors by density-gradient centrifugation using lymphocyte separation liquid (Nycomed AS, Oslo, Norway) and cultivated in the complete medium of RPMI-1640 containing 10\% FBS. The blood donors were healthy individuals who provided informed consent.

Cell proliferation assay. Cell viability was performed by the CCK- 8 assay (Dojindo Laboratories, Kumamoto, Japan). Briefly, the exponentially growing cells (1E4 cells/well) were seeded in a 96-well flat bottom microtiter plate and treated with BD926 for 24 or $48 \mathrm{~h}$. Then, $20 \mu 1$ of CCK- 8 solution was added to each well and incubated for another $4 \mathrm{~h}$ at $37^{\circ} \mathrm{C}$. The absorbance of each well was measured with Spectra microplate spectrophotometer (BioTek Instruments, Winooski, VT, USA) at $450 \mathrm{~nm}$ wavelength and the median inhibitory concentration $\left(\mathrm{IC}_{50}\right)$ was calculated with the GraphPad Prism software. Three replicate wells were used for each analysis. The results were obtained from three separate experiments.

Cell cycle analysis. After treated with BD926 for $12 \mathrm{~h}$, the cells were harvested and washed briefly in cold PBS, then fixed in 75\% ice-cold ethanol overnight. The samples were concentrated after removal of ethanol. Propidium iodide (PI) staining solution (1\% Triton X-100, 0.01\% RNase, $0.05 \%$ PI) (Sigma-Aldrich, St. Louis, MO, USA) was added to the samples to stain cellular DNA at $4^{\circ} \mathrm{C}$ for $30 \mathrm{~min}$ in dark. The cell cycle distribution was measured and analyzed by flow cytometry (FCM) (BD FACS Accuri C6; BD Biosciences, San Jose, CA USA).

Morphological observation under phase contrast microscope. Ramos cells were seeded in 12-well plates and treated with BD926 for $24 \mathrm{~h}$. Then, the morphology of Ramos cells were 
observed under a phase contrast microscope (Olympus, Tokyo, Japan).

Apoptosis analysis by flow cytometry. Annexin V-FITC/PI apoptosis detection kit (Roche Diagnostics, Indianapolis, IN, USA) was carried out to detect the apoptotic cells. Briefly, after treated with different concentrations of BD926 for $12 \mathrm{~h}$, Ramos cells were harvested and washed with cold PBS. The cells were then stained with Annexin V-FITC/PI then were analyzed by FCM.

Mitochondrial membrane potential testing. JC-1 is a fluorescent probe for the detection of mitochondrial membrane potential (MMP). After treated with BD926 for $12 \mathrm{~h}$, changes in mitochondrial transmembrane potential $(\Delta \Psi \mathrm{m})$ were evaluated by staining cells with JC-1 (Roche Diagnostics). Cell culture and drug treatment were done as described above. The harvested Ramos cells were washed with cold PBS, incubated with JC-1 $(5 \mathrm{mg} / \mathrm{ml})$ at $37^{\circ} \mathrm{C}$ for $30 \mathrm{~min}$ in the dark, then measured by FCM.

Cytochrome c detecting. Ramos cells were treated with BD926 for $12 \mathrm{~h}$, washed with PBS, fixed (BD ${ }^{\mathrm{TM}}$ Phosflow Fix Buffer) and permeabilized (BD Phosflow ${ }^{\mathrm{TM}}$ Perm Buffer), then stained with anti-cytochrome c/FITC (BD Biosciences) antibody. The resistance type with FITC labeled rat $\lg \mathrm{G} 0 / \mathrm{G} 1$ antibodies were used for comparison. FCM was used to detect cytochrome $c$ content of the cytoplasm in Ramos cells.

Western blot analysis. After treated with BD926 for $24 \mathrm{~h}$, Ramos cells were lysed in RIPA buffer (Bioteke Corp., Beijing, China) and the lysate was centrifuged at $13,000 \mathrm{x} g$ at $4{ }^{\circ} \mathrm{C}$ for $15 \mathrm{~min}$. The supernatant was harvested and the protein concentration was measured by BCA method (Bioteke). Equal amounts of total proteins were subjected to $12 \%$ SDS-PAGE and transferred onto polyvinylidene fluoride membranes (Millipore Corp., Bedford, MA, USA). After electrophoresis, the membranes were blocked at room temperature for $1.5 \mathrm{~h}$ and incubated in the respective primary antibodies at $4^{\circ} \mathrm{C}$ overnight, then the bound antibodies were detected with horseradish peroxidase (HRP)-conjugated secondary antibody (Cell Signaling Technology, Danvers, MA, USA). The immunostaining signal was visualized by enhanced chemiluminescence (Millipore).

Real-time quantitative RT-PCR. Total RNA was extracted from the treatment cells using the acid guanidinium thiocyanate-phenol-chloroform method (TRIzol; Takara Bio, Dalian, China), and cDNAs were synthesized with the PrimeScript ${ }^{\mathrm{TM}}$ RT reagent kit (Takara Bio). Quantitative PCR was performed using the Bio-Rad CFX96 Real-Time PCR detection system with the TaqMan probe method (Roche Diagnostics). The primers are 5'-cagatgaaaatgggggtaccta-3' (F) and 5'-tcaagagt ggtgaagatttttgat-3' (R) for CHOP; 5'-agctgtagcgtatggtgctg-3' (F) and 5'-aaggggacatacatcaagcagt-3' (R) for GRP78.

The mRNA expression levels were calculated with the $2^{-\triangle \Delta C T}$ method and expressed in relative quantification units. A control without cDNA was run in parallel with each assay. Each reaction was amplified in triplicate, and relative mRNA levels were normalized to GAPDH.
ROS measurement. To assess the generation of ROS, BD926-treated cells $\left(1 \times 10^{5}\right)$ were incubated with $10 \mu \mathrm{M}$ 2,7-dichlorofluorescein diacetate (DCFH-DA). Within the cells, DCFH-DA is converted to DCFH, which can be oxidized to the fluorescent compound DCF in the presence of ROS. Cell culture and drug treatment were done as described above, and Ramos cells were washed with PBS after incubated with DCFH-DA (Bi Yun Tian Corp., Kuaidamao, China) at $25^{\circ} \mathrm{C}$ for $30 \mathrm{~min}$ in the dark. Then the expression of cell fluorescence signal was detected by FCM.

Statistical analysis. The results of the statistical analysis used GraphPad Prism 5 software with one-way ANOVA and Dunnett's test, analysis, comparison between group differences. Descriptive statistics were calculated with mean \pm standard deviation, and $\mathrm{P}<0.05$ showed a statistically significant difference.

\section{Results}

BD926 inhibits the proliferation of tumor cells. In order to confirm the anticancer activity of BD926 in cell models, we examined the growth inhibition effect of BD926 on tumor cell lines including B lymphoma cells, T lymphoma cells and myeloma cells. Cells were treated with BD926 for $24 \mathrm{~h}$ and then the cell viability was assayed by CCK- 8 . The results showed that BD926 significantly inhibited the cell proliferation with $\mathrm{IC}_{50}$ from 6.0 to $42.2 \mu \mathrm{M}$ depending on different tumor cell lines (Fig. 1B-D).

The present study aimed to clarify the anticancer effect and mechanism of BD926 based on Ramos cells. The result showed, BD926 exhibited a significant cell proliferation inhibition with $\mathrm{IC}_{50} \sim 3 \mu \mathrm{M}$ for $48 \mathrm{~h}$ and $10.9 \mu \mathrm{M}$ for $24 \mathrm{~h}$ in Ramos cells (Fig. 1E). This indicated that BD926 treatment decreased the viability of Ramos cells in a time- and dose-dependent manner. The $50 \%$ inhibition concentration of cytotoxicity $\left(\mathrm{CC}_{50}\right)$ of BD926 in resting human PBMC was as high as $200 \mu \mathrm{M}$ for $48 \mathrm{~h}$ (Fig. 1F). This result suggested that BD926 had almost no cytotoxic effect on resting human PBMC under the effective inhibition concentration of tumor cells, which indicated that BD926 could effectively inhibit tumor proliferation and exhibited low toxicity in PBMC.

BD926 induces Ramos cell cycle arrest at G0/G1 phase. Because the proliferation of Ramos cells was suppressed by BD926, we determined how BD926 blocks the cell cycle progression by FCM analysis. The Ramos cells were treated by BD926 for $12 \mathrm{~h}$, the cellular DNA was stained with PI and analysed via FCM. The results are shown in Fig. 2. A dosedependent increase in the cell population of G0/G1 phase was observed. BD926 treatment increased the percentage of G0/G1 cells from $34.0 \%$ in non-treated group to $52.4 \%$ in $20 \mu \mathrm{M}$ BD926-treated group in Ramos cells. The results indicated that BD926 induced Ramos cell cycle arrest at G0/G1 phase.

BD926 induces apoptosis of Ramos cells. To obtain BD926-induced apoptosis information of Ramos cells, cell morphological changes were observed by phase contrast microscopy. BD926 induced significant apoptotic morphological changes in Ramos cells. Cells treated with BD926 became 

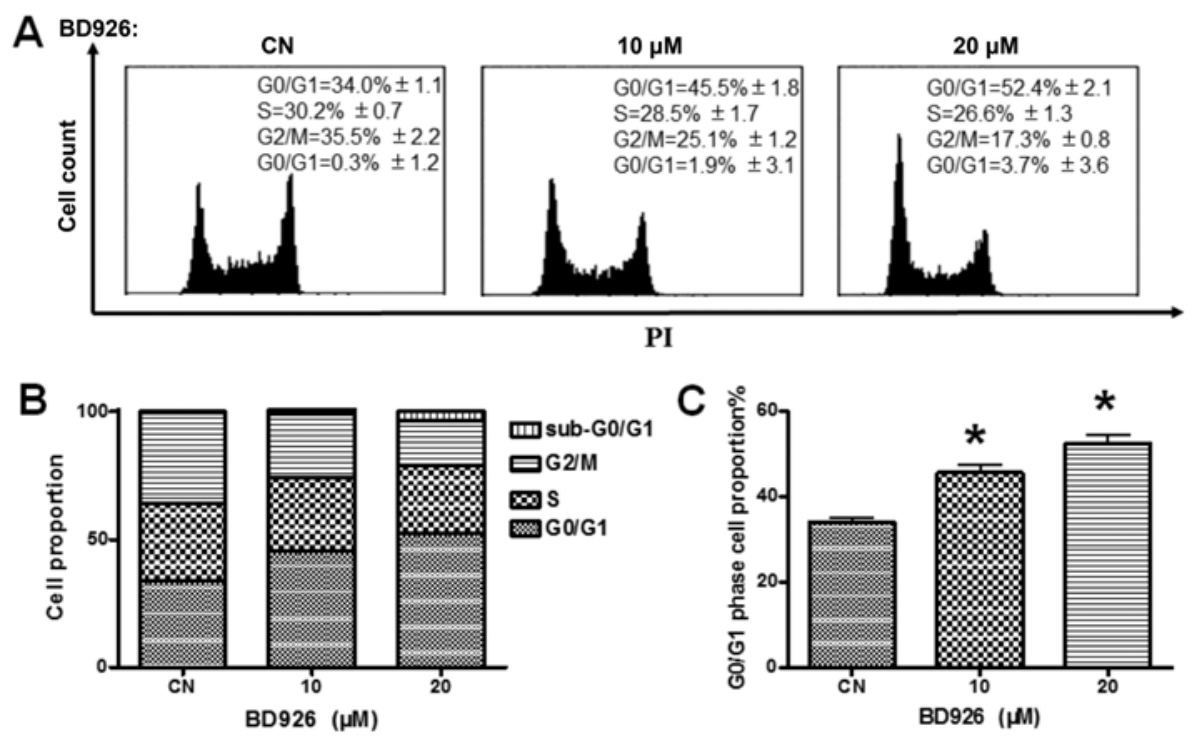

Figure 2. BD926 induces Ramos cell cycle arrest at G0/G1 phase. Ramos cells $\left(5 \times 10^{5}\right)$ were treated by varying concentrations of BD926 (20, 10 and $\left.0 \mu \mathrm{M}\right)$ for $12 \mathrm{~h}$, then the cells were washed, fixed, stained with PI, and analyzed for cellular DNA content by FCM. (A) The cell cycle of Ramos analyzed by FCM software in presence or absence of BD926. (B) The percentage of the cell cycle were analyzed with GraphPad Prism software. (C) The G0/G1 stage population was evaluated by histogram analyses. Concentration-response columns were analyzed with the GraphPad Prism software. Comparisons between groups and within groups were carried out by one-way analysis of variance (ANOVA) and ${ }^{*} \mathrm{P}<0.05$ was considered significant compared with the control.
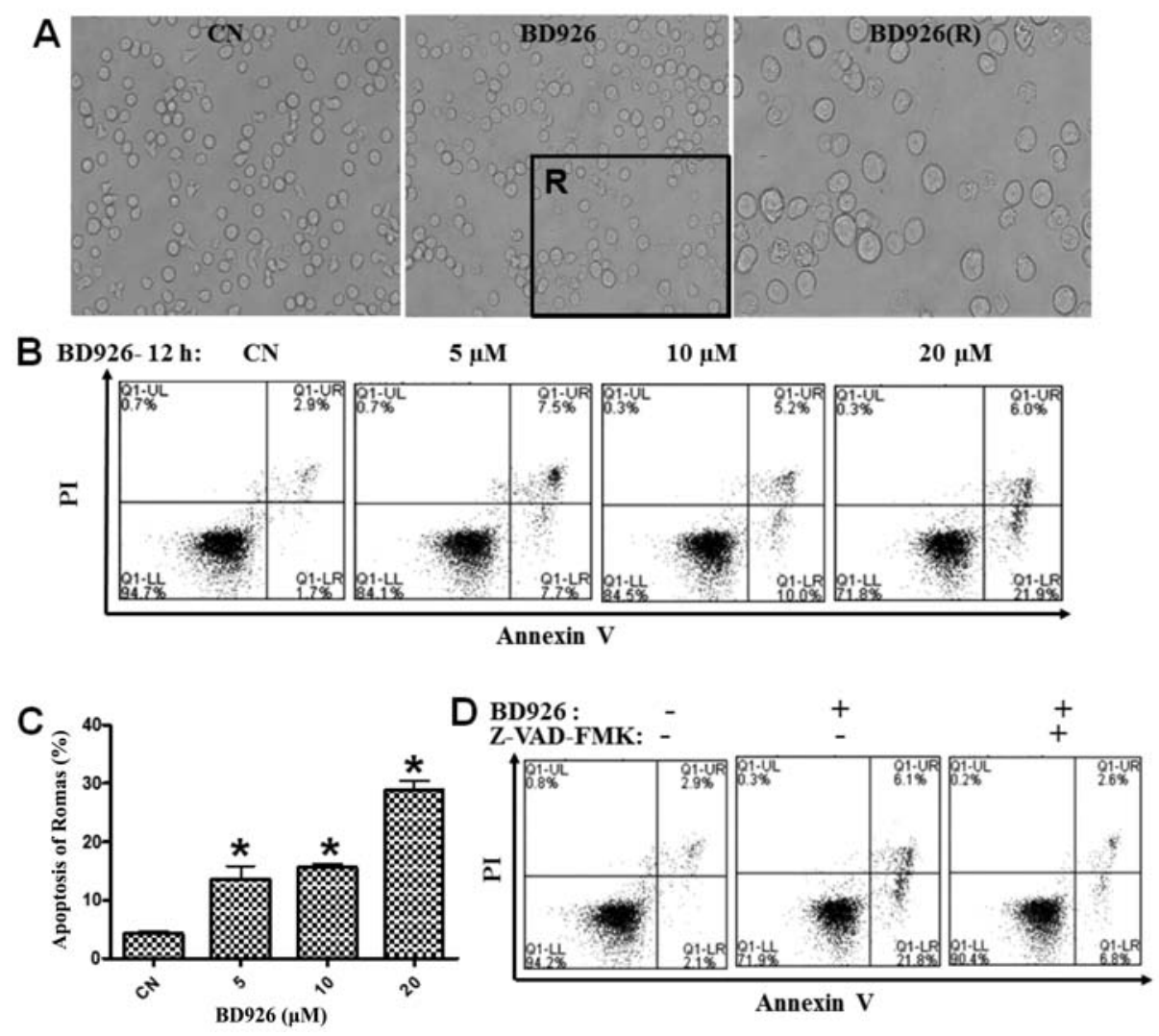

Figure 3. BD926 induces Ramos cell apoptosis. (A) Effects of BD926 on cell viability and apoptosis in Ramos cells were determined by microscopy. (B) Apoptosis effects of BD926 in Ramos cells were determined by dual staining with Annexin V-FITC and PI. The normal cells are Annexin V/PI- (Q1-LL), cells in early apoptosis are Annexin $\mathrm{V}^{+} / \mathrm{PI}^{-}(\mathrm{Q} 1-\mathrm{LR})$, cells in late apoptosis are Annexin $\mathrm{V}^{+} / \mathrm{PI}^{+}(\mathrm{Q} 1-\mathrm{UR})$ and necrosis cells are Annexin $\mathrm{V}^{-} / \mathrm{PI}^{+}\left(\mathrm{Q} 1-\mathrm{UL}^{2}\right.$ ). (C) The population of apoptotic cells (Q1-LR and Q1-UR) were evaluated by histogram analyses and ${ }^{*} \mathrm{P}<0.05$ was considered significant compared with the control. (D) Apoptosis of Ramos depended on caspase. Compared with BD926 treatment alone, treatment with $20 \mu \mathrm{M}$ BD926 combined with 20 mM Z-VAD-FMK decreased the percentage of apoptotic cells from 27.9 to $9.4 \%$.

deformed, smaller, cell membrane integrity and foaming phenomenon was seen along with apoptotic bodies (Fig. 3A).
To confirm this cell death, we also used Annexin V-FITC and PI fluorescence staining to detect the apoptosis of Ramos 


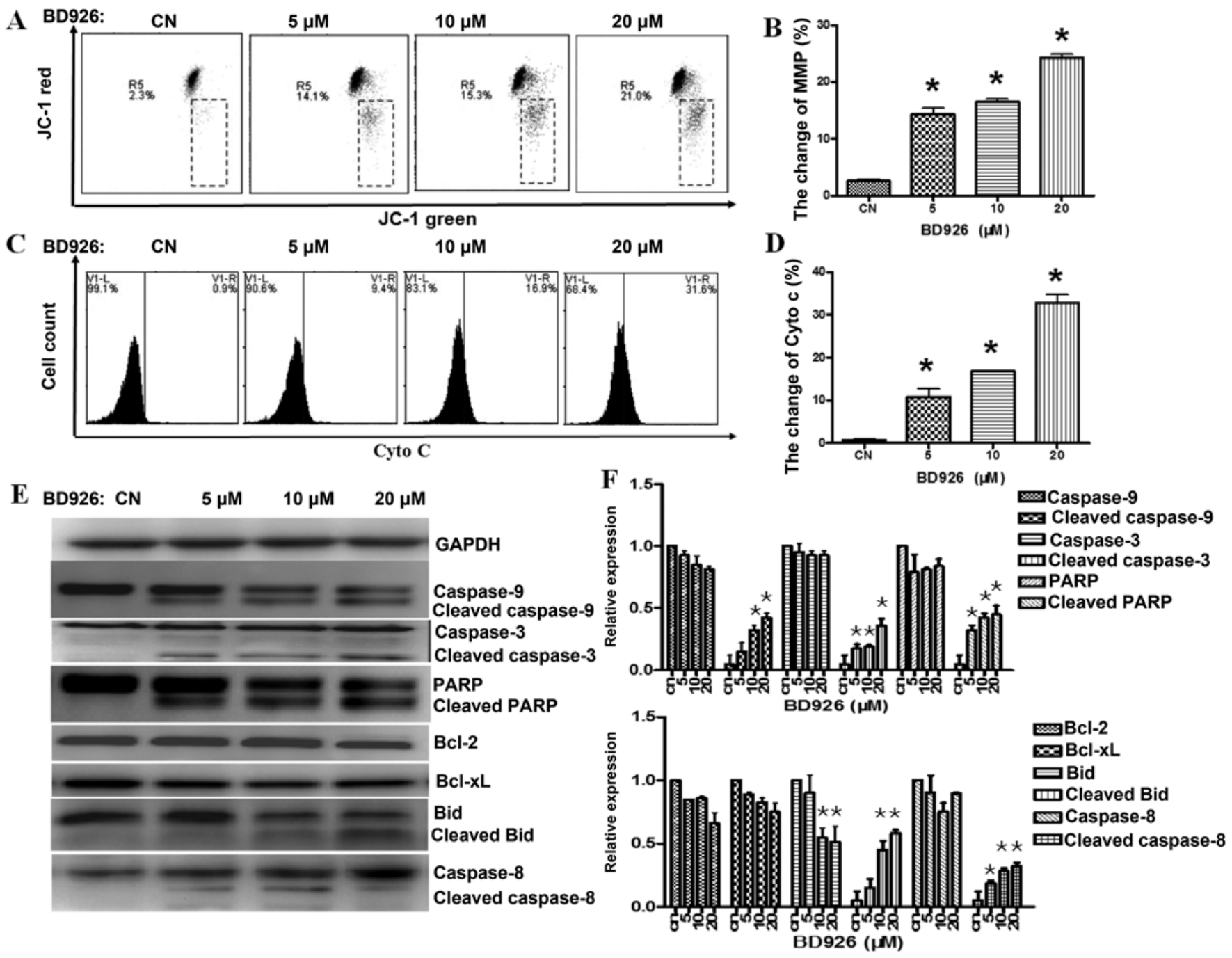

Figure 4. BD926 induces Ramos cell apoptosis by mitochondrial pathway. (A) Changes of MMP in Ramos cells were detected by JC-1. Data were analyzed using FCM software by measuring both green $(530 \pm 30 \mathrm{~nm}$; FL-1) and red ( $585 \pm 40 \mathrm{~nm}$; FL-2) JC-1 fluorescence. MMP loss was observed by a decrease in JC-1 red fluorescence and an increase in JC-1 green fluorescence. At least 5000 cells were collected and counted per sample. (B) The changes of MMP in Ramos cells were evaluated by histogram analyses. (C) The release of cytochrome $c$ in Ramos cells was analyzed using FCM. (D) The changes of cytochrome $c$ in Ramos cells were evaluated by histogram analyses. (E) The apoptotic protein expression was detected by western blot analysis. Ramos cells were treated with BD926 for $24 \mathrm{~h}$, harvested and total protein lysate was subjected to western blot analysis using antibody against caspase-3, caspase-9, caspase-8, Bid, Bcl-2, Bcl-xl, PARP, or GAPDH. (F) The apoptotic protein expression was evaluated by histogram analyses. ${ }^{*} \mathrm{P}<0.05$ was considered significant compared with the control.

cells by FCM. A dose-dependent increase in the population of apoptotic cells was observed, and the percentage of apoptotic cells reached $27.9 \%$ at the concentration of $20 \mu \mathrm{M}$ BD926 compared with $4.6 \%$ in the negative control (Fig. 3). To explore whether BD926-induced apoptosis was specifically associated with caspase activation, we examined whether Z-VAD-FMK (a general caspase inhibitor) could affect apoptosis. As shown in Fig. 3D, treatment with $20 \mu \mathrm{M}$ BD926 combined with $20 \mathrm{mM}$ Z-VAD-FMK decreased the percentage of apoptotic cells compared with BD926 treatment alone.

BD926 induces the Ramos cell apoptosis via mitochondrial pathway

Effect of BD926 on mitochondrial membrane potential $(M M P)$. Mitochondria are involved in the regulation of apoptotic cell death (13), and the change of mitochondrial membrane potential $(\Delta \Psi \mathrm{m})$ is known to be one of the important factors for mitochondrial dysfunction. Therefore, we examined $\Delta \Psi \mathrm{m}$ with JC-1 staining by FCM, on account of the dissipated MMP preventing the accumulation of JC-1 in the mitochondria, and made a shift from red (JC-1 aggregates) to green fluorescence (JC-1 monomers). As shown in Fig. 4A, BD926 depolarized the MMP in a dose-dependent manner. In addition, BD926 at a concentration of $20 \mu \mathrm{M}$ induced $21.0 \%$ decrease of $\Delta \Psi \mathrm{m}$ in Ramos cells compared with $2.3 \%$ in the negative control.

Effect of BD926 on cytochrome c. A limited step in the intrinsic apoptotic pathway is related to the release of cytochrome $c$ from mitochondria into the cytosol. To observe the change in Ramos cells, anti-cytochrome $c$ /FITC antibody was used. As shown in Fig. 4C, BD926 induced the release of cytochrome $c$ in dose-dependent manner. BD926 at a concentration of $20 \mu \mathrm{M}$ induced the release of cytochrome $c$ reaching $31.6 \%$ in Ramos cells compared with $0.9 \%$ in the negative control. 

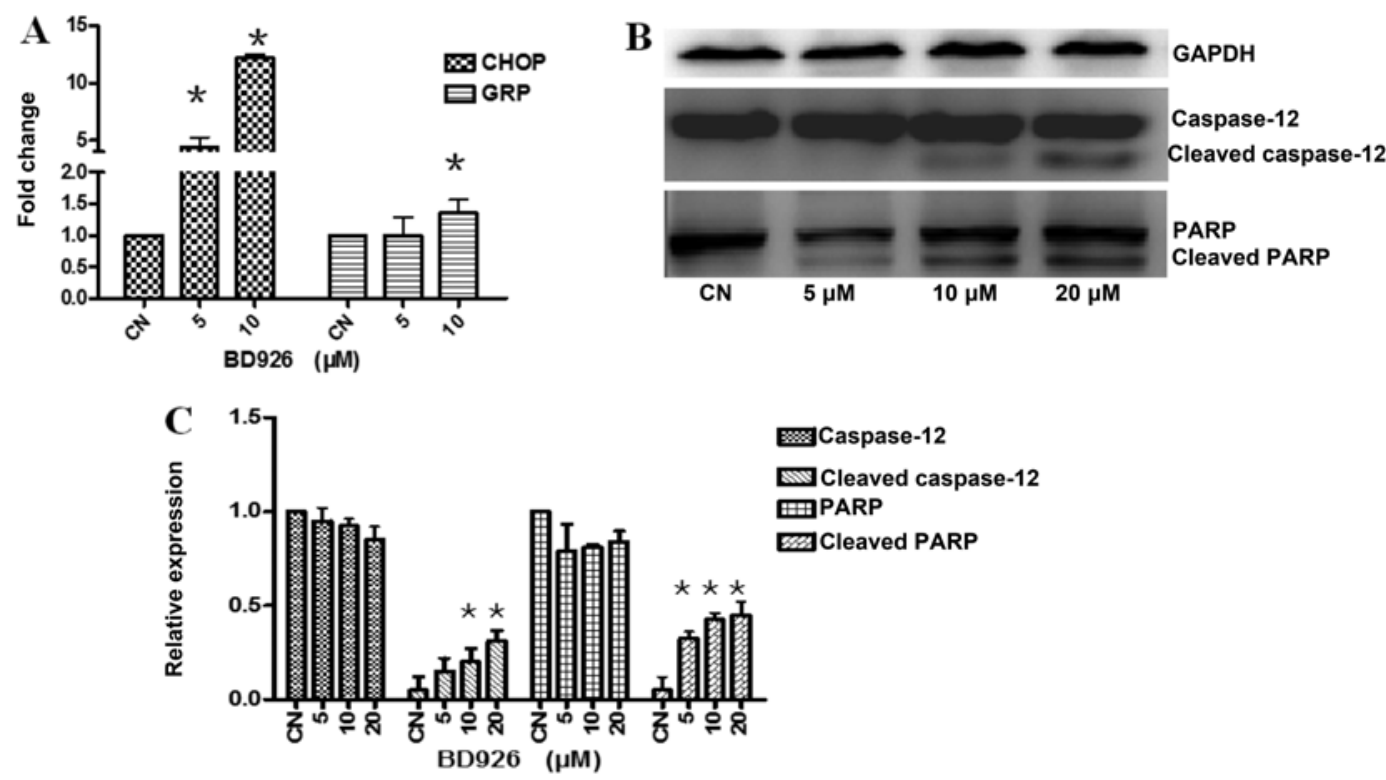

Figure 5. BD926 induces Ramos cell apoptosis by endoplasmic reticulum pathways. (A) The mRNA expression of apoptosis proteins were detected by quantitative PCR. Ramos cells were treated with BD926 $(10,5$ and $0 \mu \mathrm{M})$ for $24 \mathrm{~h}$ and the mRNA was subjected to Q-PCR analysis involving GRP78, CHOP and GAPDH. (B) The apoptotic protein expression was detected by western blot analysis. (C) The apoptotic protein expression was evaluated by histogram analyses. Concentration-response columns were analyzed with GraphPad Prism software. ${ }^{*} \mathrm{P}<0.05$ was considered significant compared with the control.

Apoptotic protein detection. To evaluate the contribution of active caspases in BD926 induced-apoptosis, we characterized the activation of caspase-9/-8/-3 and PARP by western blot analysis. As shown in Fig. 4E, a decrease in procaspases-9/-8/-3 and an increase in the levels of their cleaved forms were observed in Ramos cells. The activation of Bid and PARP protein were also observed, but the change of $\mathrm{Bcl}-2$ and Bcl-xl were not significant.

Effect of BD926 on the endoplasmic reticulum apoptosis pathway. Endoplasmic reticulum (ER) stress have shown to be involved in the cell apoptosis $(14,15)$. To confirm whether BD926 induced apoptosis by ER stress signaling, we examined the mRNA transcription of the markers in ER apoptosis pathway using real-time reverse transcription PCR. As shown in Fig. 5A, BD926-treated cells significantly increased the mRNA expression levels of ER stress-related molecules, including glucose regulated protein 78 (Grp78) which is a major protective player of the unfolded protein response (UPR) and pro-apoptotic transcriptional regulator C/EBP homologous protein GADD153/CHOP.

Caspase-12, an ER resident caspase, is localized on the ER and activated during apoptosis induced by ER stress (16). Different from caspase-7/-8/-9, caspase-12 is a specific medium which associated with ER stress-induced apoptosis (17). As the results showed, BD926 induced the activation of caspase-12 and the cleaved caspase-12 expression levels were significantly increased by BD926 (Fig. 5B). Moreover, the poly(ADP-ribose) polymerase (PARP) cleavage was also increased significantly. These results indicated that BD926 induced ER stress-associated apoptosis.

Reactive oxygen species (ROS) plays a crucial role in BD926-induced apoptosis. ROS is a mediator of intracellular signaling cascades. The excessive generation of ROS can induce oxidative stress, loss of cell functioning and apoptosis (18). To confirm whether BD926-induced apoptosis was triggered by ROS accumulation, the intracellular ROS level was measured using the ROS-detecting fluorescence dye DCFH-DA. As shown in Fig. 6A, according to the ratio of DCF-positive cells we judged that the level of ROS was significantly increased in the cells treated with BD926. In addition, BD926 at a concentration of $20 \mu \mathrm{M}$ induced 20.7\% DCF-positive cells in Ramos cells compared with $1.4 \%$ in the negative control. We also found ROS inhibitor NAC attenuated BD926-induced apoptosis (Fig. 6C). This finding suggested that ROS mediated BD926-induced apoptosis.

\section{Discussion}

The chemotherapeutic agents enhancing oxidative stress are toxic to the cancer cells because they are involved in the biological processes including cell cycle arrest, DNA repair and apoptosis. ROS is a well known mediator of intracellular signaling of cascades. The excessive generation of ROS can induce oxidative stress, loss of cell functioning and apoptosis (14). It is also reported that ROS accumulation could lead to mitochondrial dysfunction via depolarizing the mitochondrial membrane potential (18). Our findings indicate that BD926 induces apoptosis in human Ramos B-lymphoma cells, accompanying with ROS generation. ROS inhibitor NAC significantly reduces the BD926-induced ROS production and attenuates the apoptosis in Ramos cells, suggesting that ROS may play a key role in BD926-induced the apoptosis of Ramos cells.

The results showed that BD926 enhanced the disruption of MMP, induced the release of cytochrome $c$, then increased the expression of cleaved-Bid, cleaved caspase-9/-3 and its downstream target cleaved-PARP leading to activation of 

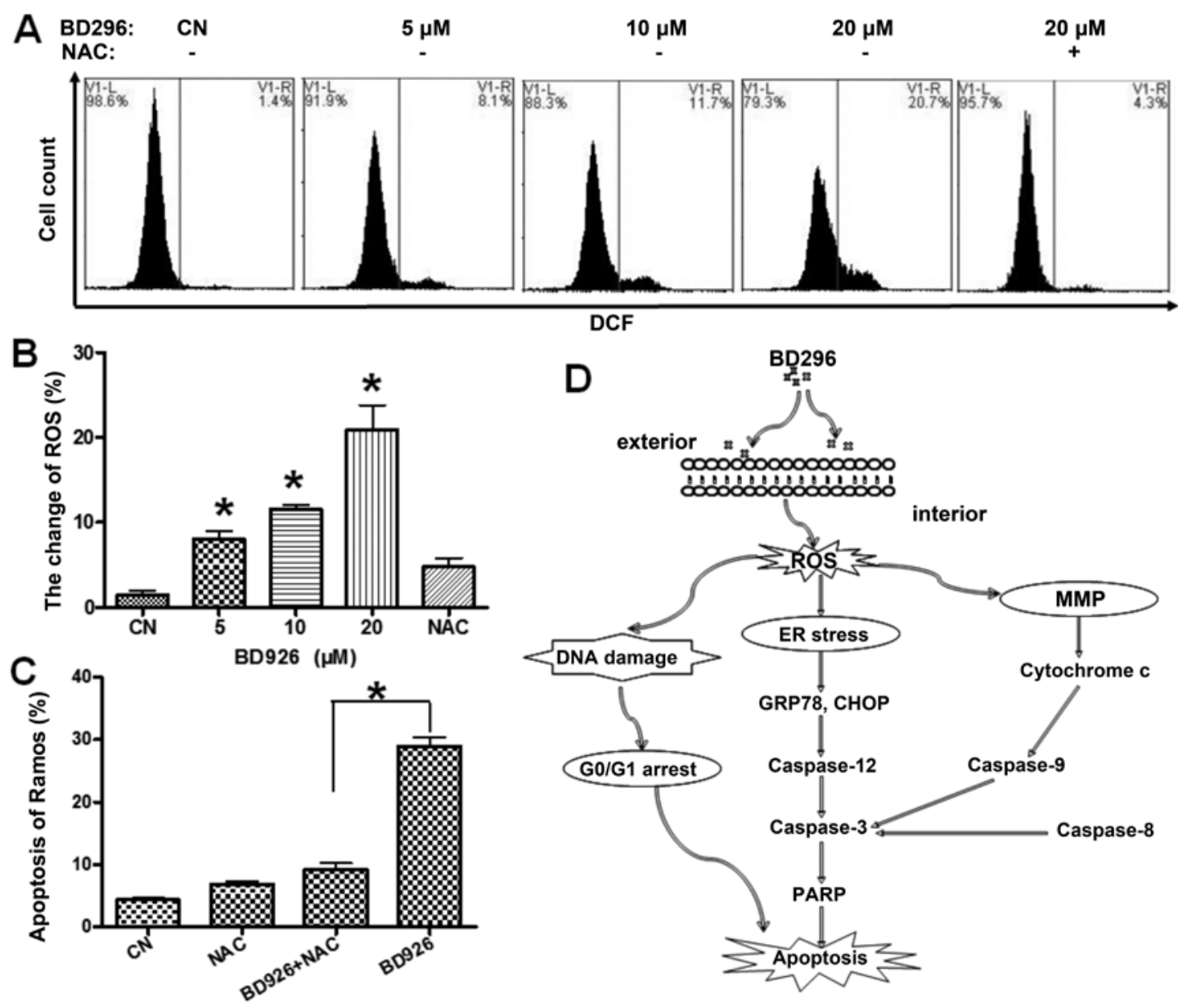

Figure 6. BD926 induces cell apoptosis via mitochondria and ER pathway mediated by ROS. (A) BD926 triggered the ROS generation in Ramos cells. Ramos cells were treated with BD926 for $12 \mathrm{~h}$, and were stained with DCFH-DA before FCM analysis. Data were analyzed using C6 FCM software by measuring DCF red (585 $\pm 40 \mathrm{~nm}$; FL-2) fluorescence. ROS was observed according to the ratio of DCF-positive cells. (B) The ROS population was evaluated by histogram analyses and ${ }^{*} \mathrm{P}<0.05$ was considered significant compared with the control. (C) NAC prevented BD926-induced apoptosis. Ramos cells pretreated for $1 \mathrm{~h}$ with $2.5 \mathrm{mM}$ NAC, then treated with BD926 for $24 \mathrm{~h}$. At the concentration of $20 \mu \mathrm{M}$ BD926, NAC prevented proportion of cell apoptosis from 27.9 to $9.2 \%$. ${ }^{*} \mathrm{P}<0.05$ was considered significant compared with BD926-treated alone. (D) The proposed model for apoptotic mechanisms activated by BD926. BD926 caused an increase of ROS in Ramos cells. Subsquently, ROS caused mitochondria-dependent apoptosis indicated by sequential events including mitochondrial depolarization, induced the release of cytochrome $c$ and activation of caspase- 9 and caspase-3. In addition, ROS triggered the ER stress by increasing the expression of apoptosis related proteins GRP78, CHOP and activated caspase-12. Moreover, ROS also caused DNA damage which lead to blocking of the cell cycle in $\mathrm{G} 0 / \mathrm{G} 1$ phase.

the mitochondrial apoptotic pathway. The increased mRNA expression of CHOP and the activated caspase-12 are also observed, which indicated that BD926 induced ER-associated apoptosis. Moreover, BD926 also resulted in cell cycle arrest at the $\mathrm{G0/G1}$ stage. In brief, we propose that BD926 activates apoptosis by increasing ROS production, which triggers the ER stress and the mitochondrial membrane dysfunction in Ramos cells, accompanied by DNA damage and cell cycle arrest (Fig. 6D).

BD926, a new water-soluble benzothiazole derivative in which the H-2 of benzothiazol was replaced by 4,5,6,7-tetrahydro-2H-indazol-3-olate group. In this study, we found that BD926 showed a growth inhibition effect against human tumor cell lines including B lymphoma cells, T lymphoma cells and myeloma cells. BD926 induced apoptosis of human Ramos B-lymphoma cells in a dose- and time-dependent manner. Importantly, BD926 had almost no cytotoxic effect on resting human PBMC under the effective inhibition concentration on tumor cells, which indicated that BD926 was able to effec- tively inhibit tumor proliferation and maintain low toxicity, a prerequisite for a lead compound.

The present study provides important insights into molecular mechanisms of the anticancer biological activities of BD926 in cell models, and the potential value of BD926 as a novel candidate antitumor drug. H-2 of benzothiazol was replaced by 4,5,6,7-tetrahydro-2H-indazol-3-olate group contributing to improve the biological activity and water solubility, which will promote the clinical application process of the benzothiazole derivatives BD926. Our findings suggest that BD926 may be a promising lead compound, design and development of new benzothiazole derivatives based on BD926 have wide reaching prospects in cancer chemotherapy.

\section{Acknowledgements}

The present study was supported by the National Natural Science Foundation of China (nos. 81301919 and 81273530), the Applied Basic Research Programs of Science and Technology 
Department of Sichuan Province (no. 2015JY0205), the Scientific Research Fund of Sichuan Provincial Education Department (no. 13ZB0220), the Research Fund of Chengdu Medical College (no. CYZ11-005), the Scientific Research Fund of Sichuan Provincial Health Department (nos. 130302 and 130298), the National Undergraduates Innovating Experimentation Project (nos. 201313705007, 201313705003 and 201413705005).

\section{References}

1. Sun D and Gündisch D: Editorial: Privileged Scaffolds in Natural Products and Drug Discovery. Curr Top Med Chem 16: 1199, 2016.

2. Keri RS, Patil MR, Patil SA and Budagumpi S: A comprehensive review in current developments of benzothiazole-based molecules in medicinal chemistry. Eur J Med Chem 89: 207-251, 2015 .

3. Sharma PC, Sinhmar A, Sharma A, Rajak H and Pathak DP: Medicinal significance of benzothiazole scaffold: An insight view. J Enzyme Inhib Med Chem 28: 240-266, 2013.

4. Gill RK, Rawal RK and Bariwal J: Recent advances in the chemistry and biology of benzothiazoles. Arch Pharm (Weinheim) 348: 155-178, 2015.

5. Singh $M$ and Singh SK: Benzothiazoles: How relevant in cancer drug design strategy? Anticancer Agents Med Chem 14: 127-146, 2014.

6. Noolvi MN, Patel HM and Kaur M: Benzothiazoles: Search for anticancer agents. Eur J Med Chem 54: 447-462, 2012.

7. Sharma PC, Bansal KK, Deep A and Pathak M: Benzothiazole derivatives as potential anti-infective agents. Curr Top Med Chem 16: 1, 2016

8. Liu Y, Yang T, Li H, Li MH, Liu J, Wang YT, Yang SX, Zheng J, Luo XY, Lai Y, et al: BD750, a benzothiazole derivative, inhibits $\mathrm{T}$ cell proliferation by affecting the JAK3/STAT5 signalling pathway. Br J Pharmacol 168: 632-643, 2013.
9. Hroch L, Aitken L, Benek O, Dolezal M, Kuca K, Gunn-Moore F and Musilek K: Benzothiazoles - scaffold of interest for CNS targeted drugs. Curr Med Chem 22: 730-747, 2015.

10. Kamal A, Syed MA and Mohammed SM: Therapeutic potential of benzothiazoles: A patent review (2010-2014). Expert Opin Ther Pat 25: 335-349, 2015.

11. Seth S: A comprehensive review on recent advances in synthesis \& pharmacotherapeutic potential of benzothiazoles. Antiinflamm Antiallergy Agents Med Chem 14: 98-112, 2015.

12. Liu Y, Lai Y, Li H, Liu J, Luo XY, Li MH, Yang T, Wang YT, Yang SX, Li LM, et al: A novel water-soluble benzothiazole derivative BD926 inhibits human activated T cell proliferation by down-regulating the STAT5 activation. Eur J Pharmacol 761: 36-43, 2015.

13. Bhola PD and Letai A: Mitochondria-judges and executioners of cell death sentences. Mol Cell 61: 695-704, 2016.

14. Farooqi AA, Li KT, Fayyaz S, Chang YT, Ismail M, Liaw CC, Yuan SS, Tang JY and Chang HW: Anticancer drugs for the modulation of endoplasmic reticulum stress and oxidative stress. Tumour Biol 36: 5743-5752, 2015.

15. Chaudhari N, Talwar P, Parimisetty A, Lefebvre d'Hellencourt C and Ravanan P: A molecular web: Endoplasmic reticulum stress, inflammation, and oxidative stress. Front Cell Neurosci 8: 213, 2014.

16. Liu D, Zhang M and Yin H: Signaling pathways involved in endoplasmic reticulum stress-induced neuronal apoptosis. Int J Neurosci 123: 155-162, 2013.

17. Szegezdi E, Fitzgerald U and Samali A: Caspase-12 and ER-stress-mediated apoptosis: The story so far. Ann NY Acad Sci 1010: 186-194, 2003.

18. Yang Y, Karakhanova S, Hartwig W, D'Haese JG, Philippov PP, Werner J and Bazhin AV: Mitochondria and mitochondrial ROS in cancer: Novel targets for anticancer therapy. J Cell Physiol: Feb 19, 2016 (Epub ahead of print). doi: 10.1002/jcp.25349. 